per day (ME/D) (OR: 2.06 [95\% Cl: $1.58-2.69]$ ), than those who were not in workers compensation program. Another study found, initial days of supply of opioids from 5 to 20 or more days was strongly associated with long term use of opioids (OR: 28.94 [95\%Cl, 23.44-35.72])5. While a study by Heins et al (2015)6 examined receiving opioids within the first month, people with back injuries were less likely to become a long-term opioid user (OR: 0.67 [95\% $\mathrm{Cl}: 0.59-0.76]$ ), while those with shoulder injury were at risk (OR: 1.29 [95\% Cl: $1.06-1.58]$ ). Conclusion: There are a number of reliable prevalence studies among workers compensation settings indicating opioid use is below 20 percent however, there remains inconsistencies when examining predictors of long term or persistent opioid use. After reviewing the literature, a validity of studies will be conducted and graded by two authors independently using a standardised checklist to complete a systematic review for publication which will assist with managing opioid use among work compensation claimants and managers.

Disclosure of Interests: None declared

DOI: 10.1136/annrheumdis-2020-eular.1548

\section{SAT0575 POSITIVE QUANTIFERON-TB GOLD TEST AND SEROCONVERSION OF QUNATIFERON-TB GOLD TEST IS ASSOCIATED WITH INCREASED RISK OF THE DEVELOPMENT OF ACTIVE TUBERCULOSIS IN PATIENTS WITH ANKYLOSING SPONDYLITIS: RESULTS FROM A REAL-WORLD DATA OVER 20 YEARS}

G. Y. Ahn ${ }^{1}$, B. S. Koo ${ }^{2}$, D. Son ${ }^{3}$, Y. J. Kim ${ }^{3}$, J. Kang ${ }^{4}$, T. H. Lee ${ }^{4}$, J. H. Kim', G. G. Song ${ }^{1}$, T. H. Kim ${ }^{4} .{ }^{1}$ Korea University Guro Hospital, Division of Rheumatology, Department of Internal Medicine, Seoul, Korea, Rep. of (South Korea); ${ }^{2}$ Inje University College of Medicine, Division of Rheumatology, Department of Internal Medicine, Seoul, Korea, Rep. of (South Korea); ${ }^{3}$ Hanyang University, Biostatistical Consulting and Research Lab, Medical Research Collaborating Center, Seoul, Korea, Rep. of (South Korea); ${ }^{4}$ Hanyang University Hospital for Rheumatic Diseases, Department of Rheumatology, Seoul, Korea, Rep. of (South Korea)

Background: With the promising efficacy and the prevalent use of anti-tumor necrosis factor-a (TNF) agents in managing AS, the risk for reactivation of latent tuberculosis infection (LTBI) still is a concern. Although guidelines include the screening and treatment of LTBI prior to the initiation of anti-TNF agent by QuantiFERON-TB Gold (QFT-G) or tuberculin test, there is a lack of evidence whether treatment of LTBI before initiation of anti-TNF agent may reduce the risk of reactivation to the same as LTBI patient without anti-TNF agent or anti-TNF agent users without LTBI. Furthermore, evidence on the need for follow-up testing and the association between seroconversion and the development of active tuberculosis is also limited.

Objectives: This study aims to investigate the real-world impact of QFT-G test on the development of active tuberculosis in patients with AS.

Methods: This retrospective study investigated 2,930 patients who had a diagnosis of AS and conducted QFT-G testing during the period of March 1998 to June 2019. 191 patients with history of treatment for LTBI or acute tuberculosis prior to the first QFT-G test and 157 patients whose hospital visits or prescription was less than 3 were excluded. Observational period was defined from the firs QFT-G test to the last hospital visit of development of active tuberculosis. The screening for development of active tuberculosis was conducted by reviewing the diagnosis, prescription of anti-tuberculosis medication, chest images and electronic medical record. Treatment of LTBI was defined when a patient was prescribed isoniazid for at least 220 for 12 months, rifampin for at least 90 days for 6 months, or concurrently prescribed isoniazid and rifampin for at least 70 days for 4 months. Wilcoxon rank-sum test, chi-square test and cox-proportional hazard analysis were performed.

Results: A total of 2687 patients (median age 32.7 years, $78.4 \%$ male, anti-TNF agent user $16.7 \%$ ) were included. Baseline QFT-G was positive in $426(20.3 \%)$ patients, and 15 active tuberculosis was observed [Incidence rate $1.5 / 1000$ person years (PY)]. Compared with baseline QFT-G (-) patients, baseline QFT-G (+) patients were older $(41.2$ years vs. 31.3 years, $p<0.001)$ and they were accompanied with more active tuberculosis (4.4/1000PY vs. 1.0/1000PY) despite the less usage of anti-TNF agents (38.5\% vs $45.8 \%, p=0.006)$. The observational period, sex, and medication utilization pattern except anti-TNF agent were similar between two groups. Multivariable analysis showed that QFT-G (+) test increases the risk of active tuberculosis more than 10 times [adjusted hazard ration 17.0, 95\% confidence interval $(\mathrm{Cl}) 5.1-56.8, \mathrm{p}<0.001$ ] after adjusting age, sex and the usage of anti-TNF agents.

Then we conducted subgroup analysis on 965 patients with baseline QFT-G (-) and follow-up QFT-G tests. Seroconversion was documented in 65 patients (6.7\%). Active tuberculosis was observed in 4 patients, and seroconversion was occurred before the development of active tuberculosis in all patients. The incidence of active tuberculosis in seroconversion patients were 10.5/1000PY.

Conclusion: QFT-G (+) and QFT-G seroconversion is associated with increased risk of the development of active tuberculosis in patients with AS.

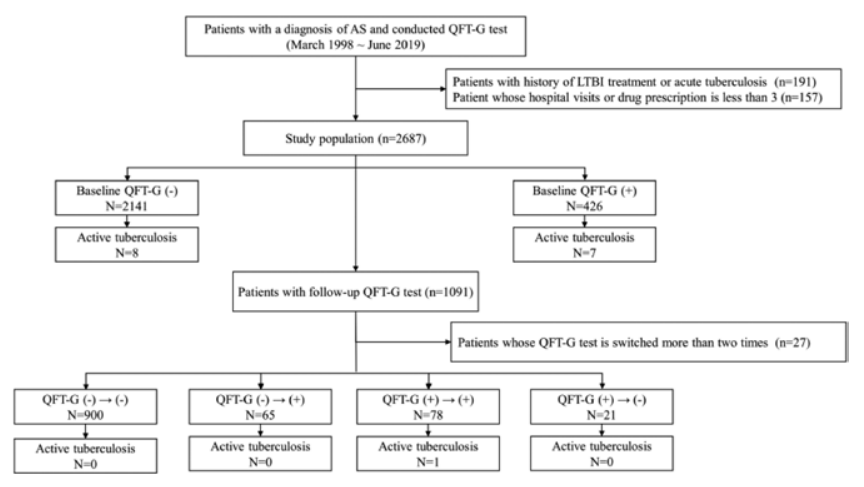

Figure 1. Overview of patient flow

Disclosure of Interests: None declared

DOI: 10.1136/annrheumdis-2020-eular.5959

SAT0576
THE PREVALENCE OF RHEUMATOID ARTHRITIS:
A SYSTEMATIC REVIEW OF POPULATION-BASED
STUDIES.

K. Almutairi ${ }^{1}$, J. Nossent ${ }^{1}$, D. Preen ${ }^{2}$, H. Keen ${ }^{1}$, C. Inderjeeth ${ }^{1} .{ }^{1}$ The University of Western Australia, School of Medicine, Perth, Australia; ${ }^{2}$ The University of Western Australia, School of Population and Global Health, Perth, Australia

Background: Rheumatoid arthritis (RA) is a heterogeneous disease with unknown aetiology (1). The reported worldwide RA prevalence varies widely (2, 3 ), and it is unclear whether this is due to inconsistencies in defining populations or methodologies used to identify RA patients $(3,4)$. Accurate RA prevalence data are required to plan preventative, diagnostic, and management strategies to address raising health care service demands and costs associated with improved lifespan and level of disability $(5,6)$.

Objectives: To estimate the prevalence of RA from international population-based studies and investigate the influence of prevalence definition, data sources, classification criteria and geographical area on RA prevalence.

Methods: A systematic review of existing literature was performed using the Joanna Briggs Institute approach for the systematic review and Preferred Reporting Items for Systematic Reviews and Meta-Analyses guidelines. A search of ProQuest, MEDLINE, Web of Science, and EMBASE was undertaken to include population-based studies investigating RA prevalence between 1980 and 2019. Results: Sixty published population-based studies met the inclusion criteria over the study period. The mean point-prevalence of RA was $0.56 \%$ (range $0.00 \%$ to $2.70 \%$ ) between 1986 and 2014 . The period-prevalence was $0.51 \%$ (range $0.05 \%$ to $1.9 \%$ ) between 1955 and 2015. RA point- and period-prevalence was higher in urban settings than rural settings, $(0.69 \%$ vs $0.48 \%)$ and $(0.54 \%$ vs $0.25 \%$ ), respectively. The mean point- and period-prevalence were $0.56 \%$ $(S D=0.52)$ and $0.57 \%(S D=0.41)$ and were lower in sampling population studies than in larger population databases studies $(0.60 \%(S D=0.27)$ and $0.44 \%(S D=$ $0.26)$ ). The highest period-prevalence of RA was observed in linked databases $(0.80 \%, S D=0.1)$ where $\mathrm{RA}$ diagnosis was validated by rheumatologists.

Conclusion: The average point- and period-prevalence of RA were $51 / 10,000$ and 56/10,000 respectively. The RA prevalence was higher in urban areas than rural areas, suggesting an impact of environmental differences. Population database studies were more consistent than sampling studies, and linked databases appeared to provide the best estimate of RA period-prevalence when rheumatologists clinically verified RA.

Table 1. The top five countries for the highest and lowest prevalence of RA in recent global estimate between 1980 and 2019.

\begin{tabular}{llc}
\hline Global prevalence of RA & Top five countries & Prevalence (\%) \\
\hline Highest & 1-Cuba & 2.70 \\
& 2-Finland & 1.90 \\
& 3-Lesotho & 1.80 \\
4-USA & 1.07 \\
Lowest & 5-Lebanon & 1.00 \\
& 1-Nigeria & 0.00 \\
& 2-Taiwan & 0.05 \\
& Taiwan & 0.10 \\
& Taiwan & 0.12 \\
& 3-Thailand & 0.12 \\
& 4-India & 0.15 \\
& 5-Philippines & 0.17 \\
\hline
\end{tabular}

